\title{
Liebe Leserin, lieber Leser
}

Mit der Einführung von Fallpauschalen in den öffentlichen Spitälern, den sogenannten Diagnosis-related Groups (DRG), steht dem schweizerischen Gesundheitswesen ein einschneidender Systemwechsel bevor. In angelsächsischen Ländern entstanden, hat das System der DRG mittlerweile auch in Deutschland Einzug gehalten. Die Schweiz ist im Vergleich dazu in Verzug, was aber die Chance beinhaltet, die Erfahrungen im Ausland in den politischen Entscheidungsprozess einzubeziehen. Richard Otto Binswanger thematisiert in seinem Beitrag Aspekte der DRGs, die für den Klinikalltag wichtig sind. ZV-Mitglied PierreFrançois Cuénoud greift das Thema im Editorial auf $(\rightarrow$ Seite 309 und $\rightarrow$ Seite 318 ).

Schlagwörter wie «Risikomanagement» und «Qualitätssicherung» sind - auch in der Medizin - in vieler Munde. Spezialisierung und Kostendruck tragen dazu bei, dass unter immer grösserem Zeitdruck immer komplexere Prozesse organisiert und abgewickelt werden müssen. Das menschliche Verhalten im Team ist dabei für die Sicherheit im System von entscheidender Bedeutung. Am Universitätsspital Basel läuft seit Ende der 1990er Jahre unter dem Namen HADmedical ein Projekt zur interdisziplinären Teamschulung, in dem Erfahrungen aus der Luft- und Raumfahrt für den medizinischen Bereich genutzt werden $(\rightarrow$ Seite 325$)$.

Die PSR/IPPNW Schweiz (Ärzte/-innen für Soziale Verantwortung/Internationale Ärzte/-innen zur Verhütung des Atomkriegs) sieht es als ihre Aufgabe an, einen Beitrag zur Prävention des Einsatzes von Nuklearwaffen zu leisten. Vom 22. bis 25. März 2007 organisiert diese Vereinigung auf dem Monte Verità oberhalb von Ascona ein Symposium mit namhaften Referenten. Im Zentrum der Veranstaltung stehen die Gefahr der nuklearen Proliferation im Nahen Osten und das Konzept einer «Atomwaffenfreien Zone im Nahen Osten» (AWFZ) $(\rightarrow$ Seite 328$)$.

Zurzeit ist in vielen Städten und Dörfern der Schweiz auf Strassen und Plätzen ein buntes Treiben zu beobachten: Das Fas- nachtsfieber hat ganze Regionen fest im Griff. Im fernen Hochland Boliviens ist Bruno Kesseli auf eine besonders wilde Spielart fasnächtlicher Tradition gestossen, bei der die Teufel in Legionsstärke aufmarschieren. Der Karneval von Oruro eine Eruption der Farben und Töne als Kehrseite der andinen Melancholie. $(\rightarrow$ Seite 340).

Sind Chirurgen attraktiver als Internisten? Und wie schneiden sie im Vergleich zu Schauspielern wie George Clooney oder Harrison Ford ab? Auch das British Medical Journal muss sich mittlerweile solch wesentlichen Fragen stellen, wie eine Publikation aus dem vergangenen Jahr zeigt. Die wissenschaftliche Studie hat Erhard Taverna dazu angeregt, sich Gedanken über die Anforderungen an einen «Mister Doc» zu machen ( $\rightarrow$ Seite 344$)$.

Die Redaktion

\section{IMPRESSUM}

\section{Redaktion}

Dr. med. et lic. phil. Bruno Kesseli (Chefredaktor)

Daniel Lüthi, FMH

Dr. med. Erhard Taverna

Redaktion Ethik

Dr. theol. Christina Aus der Au Prof. Dr. med. Lazare Benaroyo Prof. Dr. med. Lazare Bena
Prof. Dr. phil., dipl. biol. Christoph Rehmann-Sutter

Redaktion Geschichte PD Dr. med. et lic. phil. Iris Ritzman Prof. Dr. med. Jean Jacques Dreifuss Dr. rer. soc. Eberhard Wolff

Managing Editor

Thomas Heuer, lic. phil

\section{Redaktionssekretariat}

Margrit Neff

Redaktionsadresse

EMH Schweizerischer Ärzteverlag AG Farnsburgerstrasse 8, 4132 Muttenz Tel. 06146785 55, Fax 0614678556 E-Mail: redaktion.saez@emh.ch Internet: www.saez.ch
Herausgeber

FMH

Verbindung der Schweizer Ärztinnen und Ärzte

Fédération des médecins suisses

Federazione dei medici svizzeri

Fwiss Medical Association

Swiss Medical Association

Elfenstrasse 18, 3000 Bern 15

E-Mail: info@fmh.ch

Internet: www.fmh.ch

Verlag

EMH Editores Medicorum Helveticorum

EMH Schweizerischer Ärzteverlag AG

EMH Editions médicales suisses SA

EMH Edizioni mediche svizzere SA

EMH Swiss Medical Publishers Ltd.

Postfach, 4010 Basel

Tel. 06146785 55, Fax 0614678556

E-Mail: verlag@emh.ch

Internet: www.emh.ch

Herstellung

Schwabe AG

Farnsburgerstrasse 8

Postfach 832, 4132 Muttenz

Tel. 06146785 85, Fax 0614678586

E-Mail: druckerei@schwabe.ch
Marketing EMH

EMH Schweizerischer Ärzteverlag AG Thomas Gierl M. A.

Leiter Marketing und Kommunikation Farnsburgerstrasse 8, 4132 Muttenz

Tel. 06146785 49, Fax 0614678556

E-Mail: tgierl@emh.ch

Inserate

EMH Schweizerischer Ärzteverlag AG

Ariane Furrer

Assistentin Inserateregi

Farnsburgerstrasse 8, 4132 Mutten

Tel. 0614678588

Fax 0614678556

E-Mail: afurrer@emh.ch

«Stellenmarkt»

EMH Schweizerischer Ärzteverlag AG

Farnsburgerstrasse 8, 4132 Muttenz

Tel. 06146785 55, Fax 0614678556

E-Mail: stellenmarkt@emh.ch

«Stellenvermittlung»

FMH Consulting Services

Stellenvermittlung

Postfach 246, 6208 Oberkirch

Tel. 04192500 77, Fax 0419210586

E-Mail: fmhstv@hin.ch

Internet: www.fmhjob.ch
Abonnemente

FMH-Mitglieder

FMH Verbindung der Schweize

Ärztinnen und Ärzte

Elfenstrasse 18,3000 Bern 15

Tel. 03135911 11, Fax 0313591112

Andere Abonnemente

EMH Schweizerischer Ärzteverlag AG Abonnemente, Postfach, 4010 Basel Tel. 06146785 75, Fax 0614678576 E-Mail: abo@emh.ch

Jahresabonnement: CHF 320.-, zuzüglich Porto

(c) 2007 by EMH Schweizerischer Ärzteverlag AG, Basel. Alle Rechte vorbehalten. Nachdruck, elektronische Wiedergabe und Übersetzung, auch auszugsweise, nur mit schriftlicher Genehmigung des Verlages gestattet.

Erscheint jeden Mittwoch

ISSN 0036-7486

ISSN 1424-4004 (Elektronische Ausg.)
Delegierte der Fachgesellschaften Allergologie und Immunologie: Prof. Dr. A. Bircher

Allgemeinmedizin: Dr. B. Kissling Anästhesiologie und Reanimation Prof. P. Ravussin

Angiologie: Dr. B. Amman-Vesti Arbeitsmedizin: Dr. B. Merz Chirurgie: Prof. Dr. M. Decurtins Dermatologie und Venerologie: PD Dr. S. Lautenschlager Endokrinologie und Diabetologie: Prof. Dr. G.A. Spinas Prof. Dr. G.A. Spinas Geriatrie: Dr. M. Conzelmann Gynäkologie und Geburtshilfe:
Hämatologie: Dr. M. Zoppi Handchirurgie: PD Dr. L. Nagy Infektologie: Prof. Dr. W. Zimmerli Innere Medizin: Dr. W. Bauer Intensivmedizin: Dr. C. Jenni Kardiologie: Prof. Dr. B. Meier Kiefer- und Gesichtschirurgie: Dr. C. Schotland

Kinder- und Jugendpsychiatrie: Dr. R. Hotz Kinderchirurgie: Dr. M. Bittel

Medizinische Genetik: Prof. Dr. P. Miny

Neonatologie: Prof. Dr. H.-U. Bucher

Nephrologie: Prof. Dr. J.-P. Guignard

Neurochirurgie: Prof. Dr. H. Landolt

Neurochirurgie: Prof. Dr. H. Lando

Neuropädiatrie: Prof. Dr. J. Lütschg

Neuroradiologie: Prof. Dr. W. Wichmann
Nuklearmedizin: Prof. Dr. I. Mülle Onkologie: PD Dr. B. Pestalozzi Ophthalmologie: Dr. A. Franceschetti ORL, Hals- und Gesichtschirurgie: Prof. Dr. J.-P. Guyot

Orthopädie: Dr. T. Böni

ädiatrie: Dr. R. Tabin

Pathologie: Prof. Dr. G. Cathomas

Pharmakologie und Toxikologie:

Dr. T. Buclin

Pharmazeutische Medizin: Dr. P. Kleist

Physikalische Medizin und Rehabilitation:

Dr. M. Weber

Plast.-Rekonstrukt. u. Ästhetische Chirurgie:

Prof. Dr. G. Pierer

Pneumologie: Prof. Dr. E. Russi
Prävention und Gesundheitswesen: Dr. C. Junker

Psychiatrie und Psychotherapie: Dr. G. Ebner

Radiologie: Prof. Dr. B. Marincek Radioonkologie: Prof. Dr. R. H. Greiner Rechtsmedizin: Prof. T. Krompecher Rheumatologie: Prof. Dr. M. Seitz Thorax-, Herz- und Gefässchirurgie: Prof. Dr. T. Carrel

Tropen- und Reisemedizin: PD Dr. C. Hatz Urologie: PD Dr. T. Zellweger 